\title{
Hypertrophie bénigne de la prostate (HBP) géante: Aspects épidémiologiques, cliniques et thérapeutiques
}

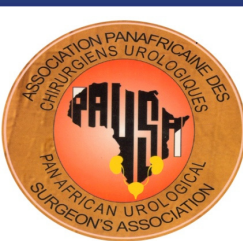

\author{
Bagayogo N.A.", Sine B., Faye M., Sarr A., Thiam A., Ndiaye M., Ndiath A., Ndour N.S., Traoré A., \\ Erradja F., Faye S.T., Sow Y., Fall B., Diao B., Ndoye A.K., Ba M.
}

Service d'Urologie-Andrologie du CHU Aristide Le Dantec, Dakar, Senegal.

\section{Résumé}

Buts: Rapporter les aspects épidémiologiques, cliniques et thérapeutiques de l'HBP géante.

Patients et méthode: Il s'agissait d'une étude rétrospective descriptive colligeant les patients ayant une prise en charge chirurgicale d'une HBP géante le 1er janvier 2012 au 30 novembre 2017. Les paramètres étudiés étaient: l'âge, les aspects cliniques et paracliniques, les données peropératoires, la durée de port de la sonde et la durée d'hospitalisation et les complications. Résultats: L'âge moyen des patients était de $70.9 \pm 9.2$ ans. La majorité des patients (13/18) avaient consulté pour une rétention complète d'urine (RCU). Le volume prostatique moyen mesuré par échographie sus pubienne était de $246.9 \pm 46.1 \mathrm{cc}$. La rétention complète d'urine (RCU) associée à une vessie de lutte (VL) et la RCU avec échec à l'épreuve d'ablation de la sonde étaient les indications opératoires les plus observées. L'adénomectomie prostatique avait été réalisée par voie transvesicale chez 15 patients et par voie rétropubienne chez trois patients. La moyenne du volume prostatique énuclée était de $148 \pm 68 \mathrm{cc}$ (40-299cc). La durée moyenne d'hospitalisation était de 6.77 jours (3-12 jours).

Conclusion: Les progrès de la chirurgie mini-invasive dans la prise en charge de l'HBP sont incontestables mais la chirurgie conventionnelle demeure le traitement de référence de cette entité.

Mots clés: Hypertrophie bénigne de la prostate; adénomectomie.

Rec,u le: 9 June 2018, Accepté le: 14 November 2018

Auteur correspondant: Ndeye Aissatou Bagayogo, Service d'Urologie-Andrologie du CHU Aristide Le Dantec, Dakar, Senegal, Email: bagaaicha12@yahoo.fr,

ISSN: 2090-2379, https://afju.journals.ekb.eg

\section{Introduction}

L'hypertrophie bénigne de la prostate (HBP), anciennement appelée adénome de la prostate, est une affection fréquente du sujet âgé. Elle est souvent responsable de symptômes qui altèrent la qualité de vie des patients et des complications qui peuvent engager le pronostic fonctionnel des reins et même le pronostic vital.

Le volume prostatique au cours de l'HBP dépasse rarement les $100 \mathrm{~g}$, ce qui ne se produit que chez $4 \%$ des hommes septuagénaires ${ }^{[1]}$.

Elle est définie comme un poids de la prostate supérieur à $200 \mathrm{~g}^{[2]}$; Ce seuil a été suggéré par les auteurs japonais du fait de la rareté de l'HBP en Asie. Il y a eu une multitude d'études sur l'HBP mais aucune ne s'est intéressée à la forme géante en Afrique. Le but de cette étude était de rapporter les aspects épidémiologiques, cliniques et thérapeutiques de l'HBP géante.

\section{Patients et méthode}

Il s'agissait d'une étude rétrospective descriptive ayant concerné les patients pris en charge chirurgicale pour HBP géante entre le 1er janvier 2012 et le 30 novembre 2017. Le caractère géant était retenu quand l'HBP avait un volume supérieur ou égal à $200 \mathrm{cc}$ à l'échographie de l'appareil urinaire.

Les patients ayant eu une adénomectomie prostatique avec un volume prostatique supérieur ou égal à $200 \mathrm{cc}$ à l'échographie de l'appareil avaient été inclus dans l'étude. Les cas de cancer de la prostate confirmé ou de tumeur prostatique suspecte avec un volume prostatique supérieur ou égal à $200 \mathrm{cc}$ à l'échographie ayant été opérés ou non avaient été exclus de l'étude.

Les paramètres étudiés étaient: l'âge des patients, les aspects cliniques et paracliniques de la tumeur, l'ECBU, la créatininémie, les données peropératoires (voie 
d'abord, la technique opératoire, volume de la tumeur prostatique énucléé), la durée du port de la sonde vésicale, de l'hospitalisation, et les complications post opératoires immédiates (Clavien-Dindo).

Les signes cliniques obstructifs et/ou irritatifs étaient regroupés sous le terme de troubles urinaires $\mathrm{du}$ bas appareil (TUBA). Le traitement médical consistait a administration du doxasosine $4 \mathrm{mg}$ ou l'alfusosine $10 \mathrm{mg}$ ( $1 \mathrm{cp} / \mathrm{j}$ le soir au coucher). L'échec du traitement médical était défini comme une absence d'amélioration des TUBA après 1 mois de traitement bien suivi. Il y avait échec de sevrage de la sonde lorsque deux tentatives d'ablation de la sonde étaient soldées par un échec après une prise d'alphabloquant au moins une semaine après la rétention complète d'urine (RCU).

Les données ont été saisies et exploitées avec le logiciel Excel 2010 et SPSS version 23.

\section{Résultats}

L'âge moyen des patients était de $70.9 \pm 9.2$ ans. La tranche d'âge la plus représentée était celle de 70 à 79 ans (Figure 1A). La majorité des patients 13/18 (72.2\%) avaient consulté pour une rétention complète d'urine (RCU). Les motifs de consultation sont rapportés dans la (Figure 1B). Les symptômes étaient associés à une hernie inguinale chez cinq patients $(27.7 \%)$. Le volume prostatique moyen mesuré par échographie sus pubienne était de $246.9 \pm 46.1 \mathrm{cc}$. La tranche de 200 à $239 \mathrm{cc}$ était la plus représentée (55.5\%). La Figure 2 rapporte la

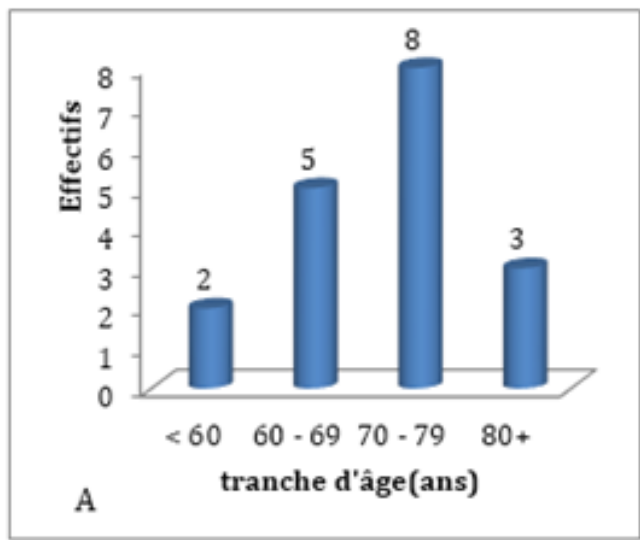

Figure 1A : Répartition des patients par tranches d'âge $(\mathrm{N}=18)$ répartition des patients selon le volume prostatique initial. La moyenne du taux de PSA était de $18.22 \pm 15.5 \mathrm{ng} / \mathrm{ml}$. Le PSA était supérieur à $10 \mathrm{ng} / \mathrm{ml}$ chez $11 / 18$ patients $(61.1 \%)$. Cependant la biopsie prostatique n'avait été réalisée que chez 8/18 patients (44.4\%). L'analyse histopathologique a révélé une hyperplasie adénomyomateuse chez tous les patients. L'examen cytobactériologique des urines (ECBU) avait mis en évidence une infection urinaire chez la moitié des patients. L'Escherichia coli et le cytrobacter freundii étaient les germes les plus incriminés. La rétention complète d'urine (RCU) associée à une vessie de lutte (VL) et la RCU avec échec à l'épreuve d'ablation de la sonde étaient les indications opératoires les plus observées. Les autres indications opératoires sont consignées dans le (tableau I). La fonction rénale était normale chez 16/18 patients $(88.9 \%)$. L'adénomectomie prostatique avait été réalisée par voie transvésicale (Hryntschak) chez 15 patients $(83.3 \%)$ et par voie retro-pubienne (Millin) chez trois patients $(16.7 \%)$. La Figure 3 montre une pièce d'adénomectomie prostatique par voie transvésicale. La moyenne du volume prostatique énucléé était de $148 \pm$ 68.5 cc $(40-299 \mathrm{cc})$. il a été noté une complication (Grade II selon Clavien-Dindo) chez deux patients. Il s'agissait d'une infection du site opératoire qui a bien évoluée sous traitement antibiotique associé à des pansements et une hémorragie ayant nécessité une prolongation de l'irrigation et une transfusion sanguine. La durée moyenne d'hospitalisation était de 6.77 jours avec des extrêmes de 3 et de 12 jours. Elle était de $6.93 \pm 1.79$ jours pour les patients opérés par voie retro-pubienne et de $4 \pm 3.61$ jours pour les patients opérés par voie transvesicale.

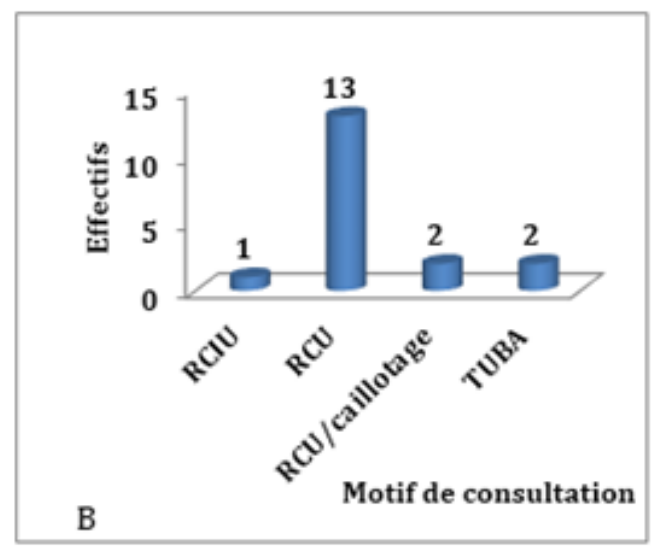

Figure 1B : Répartition des patients selon le motif de consultation $(\mathrm{N}=18)$

RCIU : rétention chronique

incomplète d'urines

RCU : rétention complète d'urines

TUBA : troubles urinaires du bas appareil 


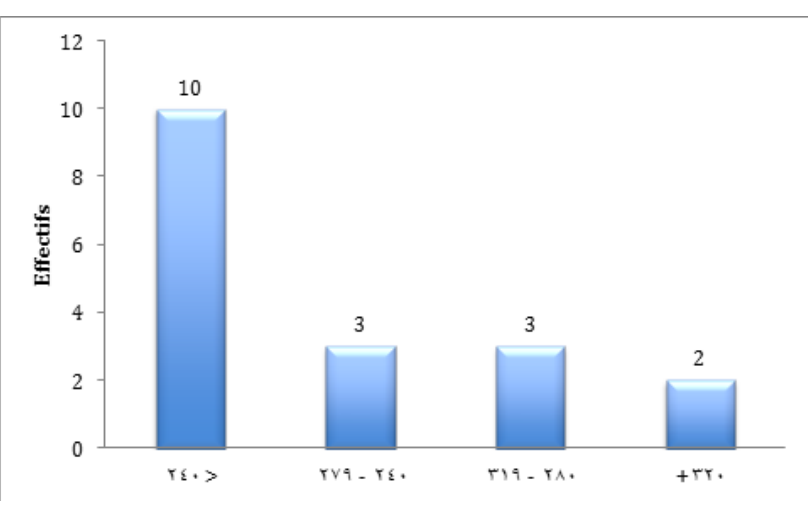

Fig. 2: Répartition des patients selon le volume prostatique à l'échographie sus pubienne $(\mathrm{N}=18)$.

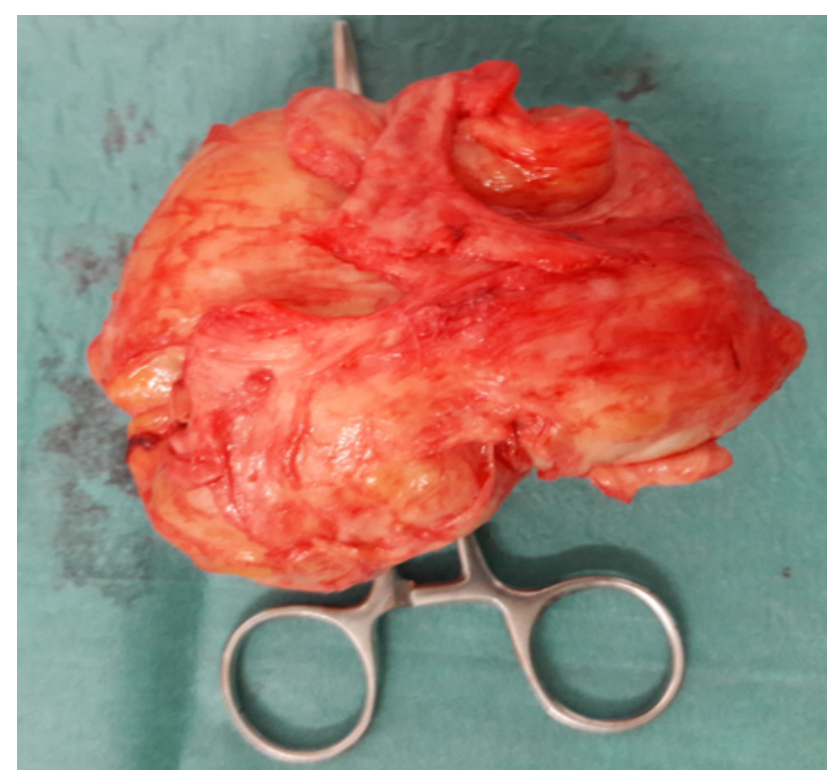

Fig. 3: Pièce d'adénomectomie prostatique transvésicale enlevée en monobloc pesant $204 \mathrm{~g}$.

Tableau I: Les différentes indications opératoires de l'HBP géante chez nos patients

\begin{tabular}{ll}
\hline Indications & Effectifs \\
\hline Echec traitement médical & 3 \\
RCU + échec sevrage sonde & 4 \\
RCU+VL & 7 \\
RCU+VL+UHN BIL & 2 \\
Vessie de lutte & 1 \\
VL+UHN bilatérale & 1 \\
Total & 18 \\
\hline
\end{tabular}

RCIU: rétention chronique incomplète d'urines

RCU: rétention complète d'urines

TUBA: troubles urinaires du bas appareil

VL: vessie de lutte; UHN BIL: Urétéro-hydronéphrose bilatérale

\section{Discussion}

L'hypertrophie bénigne de la prostate (HBP) est une affection qui atteint plus de $50 \%$ des hommes plus de 60 ans $^{[1]}$ et est le plus fréquent néoplasme bénin chez les hommes. Cette affection a gagné encore plus de notoriété en raison $\mathrm{du}$ vieillissement population en occident et l'augmentation de l'espérance de vie dans les pays du sud. La moyenne d'âge des patients était de $70.9 \pm 9.2$ ans et la tranche d'âge la plus représentée était celle de 70 à 79 ans. Ahmed Gadam et al. ${ }^{[3]}$ ont rapporté des résultats similaires dans une étude menée sur une décennie au Nigeria en mettant en évidence un âge moyen de $69.1 \pm 10.9$ ans inférieur et une tranche d'âge de 70-79 ans plus fréquente. Cette différence entre les moyennes d'âge pourrait bien faire penser que même si c'est prouvé que la prostate augmentait à partir de 50 ans, cette augmentation n'était pas proportionnelle à l'âge. Il n y a pas eu d'études sur l'existence et la physiopathologie de l'augmentation importante du volume de la prostate chez certains patients. La genèse de l'HBP géante n'est pas connue ; cependant, une surexpression exagérée des facteurs de croissance combinée à l'absence ou la réduction de facteurs inhibiteurs a été proposée comme mécanismes possibles. La mutation de certains proto-oncogènes tels que Ras et c-erbB-2 peut également être impliquée, en développant un signal de prolifération cellulaire continue ou la perte d'influence du gène suppresseur p53 à travers sa mutation ou la suppression, ce qui permettrait une anomalie de la prolifération cellulaire ${ }^{[4]}$.

La majorité de nos patients (13/18) avaient consulté pour une rétention complète d'urine (RCU). La survenue d'une RCU au cours d'une HBP constitue un tournant dans l'évolution de la maladie et c'est ce qui pousse le patient à consulter. Par ailleurs les autres motifs de consultation sont constitués essentiellement des rétentions incomplètes d'urines, des hématuries, une dysurie et une pollakiurie nocturne invalidante. Cependant, les motifs de consultation dans nos régions sont essentiellement des complications et contrastent avec ceux des pays développés où les TUBA prédominent ${ }^{[5,6]}$. Il est connu qu'il y a une absence de corrélation anatomo-clinique entre le volume et la symptomatologie qui en résulte ${ }^{[7]}$. Par contre la composante histologique dominante est déterminante. Robert et $a l .{ }^{[8,9]}$ ont rapporté une association entre l'intensité de l'inflammation histologique et les paramètres de l'HBP relatifs au volume de la prostate, au score IPSS et au type de chirurgie.

Dans notre série, les symptômes étaient associés à une hernie inguinale chez cinq patients. L'association entre hernie et HBP est fréquente. Cependant il est toujours difficile de dire si la hernie est une complication de l'HBP ou si elle est liée à une faiblesse pariétale comme ce fut le cas le plus souvent chez les sujets âgés. L'augmentation de volume de la prostate et la faiblesse musculaire sont deux situations fréquentes chez le sujet âgé. De plus, la dysurie 
secondaire à la dysectasie du col vésical se manifeste par une difficulté à vider ce qui oblige le patient à pousser lors des mictions favorisant ainsi la survenue d'une hernie inguinale ${ }^{[10]}$. Du fait de la fréquence de cette association, la recherche des TUBA chez un patient consultant pour une tuméfaction inguinale devient systématique à fortiori chez un patient de plus de 50 ans.

Le volume prostatique moyen mesuré par échographie sus pubienne était de $246.9 \pm 46.1 \mathrm{cc}$. Dans la littérature, seulement des cas isolés d'HBP géantes ont été rapportés ${ }^{[11-13]}$. Dans notre série le plus grand volume de prostate était de $345 \mathrm{cc}$.

Le PSA était supérieur à $10 \mathrm{ng} / \mathrm{ml}$ chez $11 / 18$ patients. Malgré l'augmentation du PSA avec le volume prostatique, un taux de PSA $>4 \mathrm{ng} / \mathrm{ml}$ est une indication de réaliser des biopsies prostatiques avec examen anatomopathologique. Le PSA est un marqueur spécifique de la glande prostatique et les facteurs favorisants son augmentation sont multiples : le cancer de la prostate, l'HBP, l'infection (prostatite), les biopsies prostatiques, l'éjaculation, l'âge, la race, le TR. La biopsie prostatique n'a été réalisée que chez 8 patients.

L'examen cytobactériologique des urines (ECBU) avait mis en évidence une infection urinaire chez la moitié des patients. L'Escherichia coli et le Cytrobacter freundii étaient les germes les plus incriminés. L'hypertrophie prostatique et la rétention d'urine qui la complique constituent deux facteurs favorisants de l'infection urinaire. L'Escherichia coli est le germe le plus responsable d'infections urinaires $^{[14]}$. Ceci est dû à la virulence du germe et à ses propriétés à se fixer et à migrer le long des voies urinaires grâce à ses pili. Eden et al..$^{[15]}$ avaient émis l'hypothèse selon laquelle la capacité de se fixer aux cellules uroépithéliales pourrait être un facteur de virulence pour les souches d'Escherichia coli qui provoquent une infection des voies urinaires symptomatiques.

La rétention complète d'urine (RCU) associée à une vessie de lutte (VL) et la RCU avec échec à l'épreuve d'ablation de la sonde étaient les indications opératoires les plus observées dans notre étude. Il n'y a pas d'indication opératoire selon le volume de la prostate. Qu'il s'agisse d'une HBP géante ou non les indications opératoires restent les mêmes. Cependant il existe des limites pour certaines techniques mini invasives quand le volume de la prostate dépasse $100 \mathrm{cc}$.

L'adénomectomie prostatique était le traitement de choix chez nos patients. La chirurgie ouverte a été longtemps préconisée pour des prostates volumineuses chez des patients en bon état général, qui peuvent supporter une laparotomie. Certes, la chirurgie conventionnelle reste le traitement de référence en cas d'HBP géante mais les progrès dans les techniques mini-invasives ont commencé à défier cette notion avec des avantages tels que des taux plus faibles de saignement et de transfusion et un séjour hospitalier plus court. C'est ainsi que Gopee et al. ${ }^{[16]}$ ont rapporté l'utilisation de l'énucléation de la prostate au laser holmium (HoLEP) dans une glande mesurant plus de $400 \mathrm{cc}$. Yun et al. et Zeng et al. ${ }^{[17,18]}$ ont respectivement rapporté l'utilisation de la voie d'abord coelioscopique pour des volumes prostatiques de 75 à $190 \mathrm{cc}$ et $500 \mathrm{cc}$. Learny et al. ${ }^{[19]}$ ont aussi utilisé avec succès l'embolisation des artères prostatiques associée á la RTUP et à la 5alpharéductase pour prendre en charge une HBP géante de $463 \mathrm{cc}$.

Par ailleurs la moyenne du volume prostatique énucléé était de $148 \pm 68.5 \mathrm{cc}(40-299 \mathrm{cc})$. Les différences observées entre les volumes mesurés à l'échographie sus pubienne et les volumes des tumeurs énucléés étaient dues au fait que l'échographie endorectale est plus performante que l'échographie sus pubienne dans l'évaluation du volume prostatique. De plus l'échographie constitue un examen opérateur dépendant. La durée moyenne d'hospitalisation dans notre série était inférieure à celles de 12.1 jours et 8 jours rapportées par Fourcade et al..$^{[20]}$ et Fall et al..$^{[10]}$. Cependant dans ces études l'abord était seulement transvésicale.

La durée d'hospitalisation était inferieure chez les patients opérés par voie rétro-pubienne du fait de l'absence d'ouverture de la vessie qui nécessite habituellement cinq jours pour cicatriser. Ceci pourrait expliquer la différence de la durée d'hospitalisation entre ces études et la nôtre. En effet dans notre étude la durée moyenne des patients opérées par voie retro-pubienne était de $6.93 \pm 1.79$ jours et celle des patients opérés par voie transvésicale était de $4 \pm 3.61$ jours. Par ailleurs la prolongation de la durée d'hospitalisation pourrait être due à la survenue de complications précoces telles que l'infection du site opératoire ou l'hémorragie. Ceci a été le cas chez un de nos patients qui avait une prolongation de l'irrigation vésicale liée à une hématurie ( 8 jours) et un autre qui a eu une suppuration de la plaie opératoire (12 jours).

\section{Conclusion}

L'HBP géante constitue une entité à connaître absolument même si ses manifestations cliniques ne diffèrent guère de celles des autres entités. Les progrès de la chirurgie mini-invasive dans la prise en charge de l'HBP sont incontestables mais la chirurgie conventionnelle demeure le traitement de référence de cette entité.

\section{Acknowledgements}

Toute notre gratitude aux Professeurs Babacar Diao, Alain Khassim Ndoye et Mamadou Ba. 


\section{Intérêts concurrents}

Les auteurs déclarent ne pas avoir de conflits d'intérêts en relation avec cet article.

\section{Ethique}

Le consentement éclairé des parents a été obtenu pour l'utilisation des images et l'exploitation du dossier médical à des fins scientifiques.

\section{Contribution Des Auteurs}

Dr Babacar Sine, Dr Maguette Faye ont activement participé à la rédaction et à la correction de l'article.

Dr Alioune Sarr, Dr Modou Ndiaye, Dr Amath Thiam, Dr Abdoulaye Ndiath, Dr Ndiaga Seck Ndour1, Dr Faddwa Erradja, Dr Aboubacar Traoré, Dr Samba Thiapato Faye et nous avons collaboré ensemble dans la prise en charge du patient, la rédaction et la correction de ce document.

Les professeurs Yaya Sow, Boubacar Fall, Babacar Diao, Alain K. Ndoye, Mamadou Ba sont les responsables d'enseignement dans notre service. Ils ont tous contribué à la rédaction et la correction de cet article.

Financement: Aucun financement

Consentement à la publication: Non applicable.

\section{Références}

1. Platz EA, Smit E, Curhan GC, et al. Prevalence of and racial/ethnic variation in lower urinary tract symptoms and noncancer prostate surgery in U.S. men. Urology 2002; 59 (6):877-83

2. Kawamura S, Takata K, Yoshida I, et al. A case of giant prostatic hypertrophy. Hinyokika Kiyo 1984; 30:1861-6

3. Ahmed Gadam I, Nuhu A, Aliyu S. Ten-year experience with open prostatectomy in maiduguri. ISRN Urol 2012;2012:406872

4. Silva-Gutierrez A, Perez-Evia CA, Alcocer-Gaxiola $\mathrm{B}$, et al. Giant prostatic hyperplasia: A case report and literature review. Rev Mex Urol 2010; 70:183-6.

5. Kurokawa K, Tamura Y, Ogura H, et al. Open surgery of elderly patients with benign prostatic hypertrophy, Acta Urol Jap 1990; 36(10):1167-72
6. Tubaro A, Carter, S., Hind A., et al. A prospective study of the safety and efficacy of suprapubic transvesical prostatectomy in patients with benign prostatic hyperplasia. J Urol 2001; 166(1);172-6.

7. Lebdai S, DescazeaudA. Prise en charge des symptômes du bas appareil urinaires liés à l'hypertrophie bénigne de prostate. Prog Urol 2014; 24 (14), 929-33.

8. Robert G, Descazeaud A, Allory Y, et al. Inflammation in prostatic tissue is associated with symptomatic BPH, IPSS and prostate volume. J Urol 2009; 181(4): 504.

9. Robert G, Descazeaud A, Nicolaïew $\mathrm{N}$, et al. Inflammation in benign prostatic hyperplasia: a 282 patients' immunohistochemical analysis. Prostate 2009; 69:1774-80

10. Fall PA, Gueye SM, Ndoye AK, et al. Mortalité et Morbidité précoces après adénomectomie prostatique par voie transvésicale. Afr J Urol 2002; 8 (1): 20-3.

11. Lacy JM, Bole R, Hendrix L, et al. Retropubic prostatectomy for giant benign prostatic hyperplasia. Can J Urol 201522 (5): 8000-2

12. Maliakal J, Mousa EE, Menon V. Giant Prostatic Hyperplasia: Fourth largest prostate reported in medical literature. Sultan Qaboos Univ Med J 2014 14 (2): e253-6

13. Dominguez A, Gual J, Munoz-Rodriguez J, et al. Giant Prostatic Hyperplasia: Case Report of $3987 \mathrm{~mL}$. Urology 2016; 88: e3-4

14. Bruyère F, Cariou G, Boiteux JP, et al. Diagnostic, traitement et suivi des infections urinaires bactériennes communautaires de l'homme et de la femme (cystite aiguë et pyélonéphrite aiguë) et de l'appareil génital de l'homme (prostatite aiguë). Prog Urol 2008; 18 (Suppl 1): 4-8.

15. Edén, CS, Jodal U, Hanson LA, et al. Variable adherence to normal human urinary-tract epithelial cells of Escherichia coli strains associated with various forms of urinary-tract infection. The Lancet 1976; 308 (7984): 490-2 
16. Gopee L, Hong MK, Pham T. Holmium laser enucleation of the prostate in a $400 \mathrm{cc}$ prostate: case report. J Endourol Case Rep 2016; 2 (1): 21-3

17. Yun HK, Kwon JB, Cho SR, et al. Early experience with laparoscopic retropubic simple prostatectomy in patients with voluminous benign prostatic hyperplasia (BPH). Kor J Urol 2010;51(5):323-9.

18. Zeng QS, Zhao YB, Wang BQ, Ying M, Hu WL. Minimally invasive simple prostatectomy for a case of giant benign prostatic hyperplasia. Asian J Androl $2017 ; 19(6): 717$
19. Learney RM, Malde S, Downes M, et al. Successful minimally-invasive management of a case of giant prostatic hypertrophy associated with recurrent nephrogenic adenoma of the prostate. BMC Urol 2013; 13 (1): 18

20. Fourcade RO, Lando Y, Teillac P. Les résultats du traitement chirurgical de l'hypertrophie bénigne de la prostate. Rapport du 87e congrès de l'Association Française d'Urologie. Prog Urol 1993; 3: 823-906 


\title{
Giant Benign prostatic hypertrophy (BPH): Epidemiological, clinical and therapeutic aspects
}

\begin{abstract}
Objectives: To report the epidemiological, clinical and therapeutic aspects of giant BPH.

Patients and Methods: This was a retrospective descriptive study of patients with surgical management of a giant BPH from January 1, 2012 to November 30, 2017. The parameters studied were: age, clinical and para-clinical aspects, intraoperative data, duration of catheterization and duration of hospitalization and complications.

Results: The average age of the patients was $70.9 \pm 9.2$ years. The majority of patients $(13 / 18)$ had consulted for complete urinary retention (CUR). The average prostatic volume measured by suprapubic ultrasonography was $246.9 \pm 46.1 \mathrm{cc}$. Complete urinary retention (CUR) associated with a control bladder (VL) and CUR with failed ablation of the probe were the most observed operative indications. Prostatectomy was performed transvesically in 15 patients and by the retro-pubic route in three patients. The average prostate volume enucleate was $148 \pm 68.5 \mathrm{cc}(40-299 \mathrm{cc})$. The average duration of hospitalization was 6.77 days (3-12 days).

Conclusion: Advances in minimally invasive surgery in the management of BPH are indisputable, but conventional surgery remains the reference treatment for this entity.
\end{abstract}

Keywords: Benign prostatic hyperplasia; prostatectomy. 